\title{
Pharmacy Vaccinations
}

\section{R. Oehlmann (Ralf Oehlmann), A. Czirfusz (Attila Czirfusz)}

SEUC PhD program in Health management and public health, Germany.

\section{E-mail address:}

ralf.oehlmann@web.de

\section{Reprint address:}

Ralf Oehlmann

SEUC PhD program in Health management and public health

Pharmacist, Abbentorswallstr. 52

28195 Bremen

Germany

Source: Clinical Social Work and Health Intervention

Volume: 12

Issue: 2

Pages: $12-16$

Cited references: 17

\section{Reviewers:}

Daria Kimuli

Catholic university of Eastern Africa, Nairobi, KE

Roberto Cauda

Institute of Infectious Diseases, Catholic University of the Sacred Heart, Rome, IT

\section{Keywords:}

Anti-vaccination Movements. Vaccinations. Pharmacists. Pandemics. Healthcare Access.

Disease T.ransmission.

\section{Publisher:}

International Society of Applied Preventive Medicine i-gap

CSWHI 2021; 12(2): 12 - 16; DOI: 10.22359/cswhi_12_2_02 (C) Clinical Social Work and Health Intervention

\section{Abstract:}

The vaccination rate is on the decline as parents avoid making frequent visits to healthcare facilities to visit doctors. The high cost of healthcare access in Germany, the United States, and other parts of the world has resulted in many people remaining under vaccinated or unvaccinated. The rate of pandemic outbreaks in Europe has highlighted the significance of improved communication and education about the safety and the efficacy of vaccinations alongside effective strategies of reducing the rate of disease transmission ${ }^{1}$. Pandemics have unprecedented impacts on the families' health and pressure on healthcare systems in Europe. During pandemics, there is widespread fear in families concerning how the pandemics will affect families and communities more especially to the compromised people in society, such as pregnant women and people with chronic illnesses. Although pharmaceutical practices are under intensive 
scrutiny, pharmacies' vaccinations comprise an important element in the healthcare system for pandemic diseases.

\section{Pharmacy vaccinations}

Throughout the history of public health, vaccinations play a critical role during an outbreak of pandemics helping to reduce transmission and eradication of pandemics. Europe has experienced many pandemics with detrimental impacts on the healthcare system and economies of many countries. During such pandemics, there is intense pressure on the existing healthcare systems, especially a shortage of critical equipment such as respirators and limitation in bed capacity during respiration related pandemics ${ }^{2}$. This makes pharmacists very essential in facilitating the supply of vaccinations during pandemics.

Pharmacies are considered to be the most accessible healthcare providers. The increasing number of pharmacies in Europe and other parts of the world makes them easily accessible by many people compared to the public and private healthcare access facilities ${ }^{3}$. Before, during, and after pandemics, the interaction between people and pharmacists is high. Pharmacists are better positioned to provide the public health with appropriate information regarding the minimization of transmissions, appropriate treatments and vaccinations. Pharmacists are central in the healthcare system in Germany and many parts of Europe. They advocate for improved and proper vaccination during the outbreak of pandemics. In some instances, community pharmacists execute programs that help to reach out and vaccinate many patients within their communities. They also recommend the diagnosis, appropriate vac- cinations, and advice on hospital admission. Pharmacists provide continuous vaccination education to members of the public, equipping them with enough information about the significance of taking vaccinations during pandemics and the benefits to the families and communities $^{4}$. Pharmacists ensure that patients complete routine vaccination programs during pandemics by being more proactive in their role.

Pharmacists play an essential role in countering anti-vaccination campaigns, which act as a major hindrance to vaccination programs. For instance, in 1998, The Lancet published a Wakefield and colleagues investigation which associated health complications such as autism to measles vaccination ${ }^{5}$. This comprised of a major anti-vaccination movement. Pharmacists at The Lancet were able to disapprove the assertions made by their colleagues in their published reports terming them as incorrect assertions. Since then, pharmacists have played a central role in measles vaccinations; eventually, measles was reduced significantly reduced and eradicated in many parts of Europe. As anti-vaccination movements rise, measles resurges in Europe.

During pandemic outbreaks, pharmacists play an essential role in ensuring that individuals identified receive timely vaccination and are isolated in good time, preventing further transmissions. This happens through close collaboration with other public health and government authorities and international health organizations such as the world health organization ${ }^{6}$. During pandemics,

\footnotetext{
${ }^{1}$ Centers for Disease Control and Prevention. (2007). Key facts about seasonal influenza (flu). The Influenza. http://www.CDC.gov/flu/key facts. htm (accessed November 28, 2008).

${ }^{2}$ Burson, R. C., Buttenheim, A. M., Armstrong, A., \& Feemster, K. A. (2016). Community pharmacies as sites of adult vaccination: A systematic review. Human vaccines \& immunotherapeutics, 12(12), 3146-3159.

${ }^{3}$ Patel, M. (2019). Increase in measles cases - United States, January 1-April 26, 2019. MMWR. Morbidity and mortality weekly report, 68 .

${ }^{4}$ Guerci, J., Campbell, C. T., \& Curtis, S. D. (2019). The Pharmacists' Role in Disease Outbreaks: Navigating the Dynamics of Uncertainty Before, During, and After Disease Outbreaks.

${ }^{5}$ Eggertson, L. (2010). The Lancet retracts 12-year-old article linking autism to MMR vaccines. Canadian Medical Association. Journal, 182(4), E199.

${ }^{6}$ Guerci, J., Campbell, C. T., \& Curtis, S. D. (2020). The Pharmacists' Role in Disease Outbreaks: Navigating the Dynamics of Uncertainty Before, During, and After Disease Outbreaks.
} 
many people feel insecure visiting healthcare facilities due to fear of contracting infections. This hinders government campaigns encouraging people to visit healthcare facilities for routine immunization and vaccinations against infectious diseases. This drops the rate of vaccination significantly. Pharmacists are vigilant learners during pandemics, which help track the performance of immunized patients while keeping track of the transmissions ${ }^{7}$. In the emergence of pandemics, pharmacists inform public health agencies for further action and vaccination programs. They also help to detect any resurgence during pandemics. Pharmacists play an essential role in educating communities on the role of vaccinations.

According to the Center for Disease Control, pharmacists comprise an essential source of vaccination information to the community members. Pharmacists frequently interact with the community. During the resurgence of the emergence of life threatening diseases and infections, vaccinations are critical in saving many lives ${ }^{8}$. For instance, in the United States in 2009, pharmacists from the 50 states were authorized to administer the influenza vaccine. Pharmacists in 46 states were allowed to administer all adult vaccines. There has been a significant rise in the number of pharmacists authorized to administer vaccinations in Europe ${ }^{9}$. For instance, the percentage of pharmacists allowed to immunize rose from $20 \%$ in 2010-2011 to $25 \%$ in $2014-2015$. The rise comprises an indication of the benefits of engaging pharmacists in vaccination programs during pandemics. Alongside the vaccination, pharmacists provide time information to the public and give reports to the public health agencies. The feedback collected from the pharmacists can be used to improve the vaccination programs making them more effective.

A meta-analysis study in 2016 indicated a rise in the number of vaccinations when the pharmacists were involved as facilitators and administrators compared to programs that failed to involve the pharmacists in vaccination programs. In other studies, Drodze et al. found that many countries in Europe were amending their legislation to allow pharmacists to administer vaccinations. After the amendment in the legislation, the percentage of immunized adults increased from $32.2 \%$ in 2003 to 40.3 in the year $2013^{10}$. In another survey in 2014 on the availability and accessibility of pharmacists administered vaccinations, one-third of the sample population during the study reported they could not have received vaccinations if the pharmacist administered vaccinations were not available. Pandemics have unprecedented impacts on the economies and healthcare systems of many countries ${ }^{11}$. This created the need for cheap and convenient vaccine administration programs. The pharmacists' vaccination administration programs are convenient and involve lower costs for the public's vulnerable members, such as those with low income.

During pandemics, the society's vulnerable members are at a bigger risk, especially people with limited income to access diagnosis and treatment. In such instances, pharmacists provide lower-cost vaccinations helping to save many lives during pandemics ${ }^{12}$. Pharmacists engage in multidisciplinary teams that help evaluate the history of patient vaccinations and provide further details on vaccination before making patient discharge.

\footnotetext{
${ }^{7}$ Patel, M. (2019). Increase in measles cases-United States, January 1-April 26, 2019. MMWR. Morbidity and mortality weekly report, 68 .

${ }^{8}$ Hussain, A., Ali, S., Ahmed, M., \& Hussain, S. (2018). The anti-vaccination movement: a regression in modern medicine. Cureus, 10(7).

${ }^{9}$ Centers for Disease Control and Prevention. (2007). Key facts about seasonal influenza (flu). The Influenza. http://www.CDC.gov/flu/key facts.htm (accessed November 28, 2008).

${ }^{10}$ Tsuyuki, R. T., Beahm, N. P., Okada, H., \& Al Hamarneh, Y. N. (2018). Pharmacists as accessible primary health care providers: review of the evidence. Canadian Pharmacists Journal/Revue des pharmaciens du Canada, 151(1), 4-5.

${ }^{11}$ Isenor, J. E., Edwards, N. T., Alia, T. A., Slayter, K. L., MacDougall, D. M., McNeil, S. A., \& Bowles, S. K. (2016). Impact of pharmacists as immunizers on vaccination rates: a systematic review and meta-analysis. Vaccine, 34(47), 5708-5723.

${ }^{12}$ Hoffman, J. (2020). Vaccine rates drop dangerously as parents avoid doctor's visits; 2020.
} 
The outbreak of COVID-19 in Europe and the world has proved pharmacists essential members of the healthcare sector. According to a study conducted in 2020 , the prevailing COVID-19 pandemic has been the major factor causing the decline of childhood vaccinations in Europe. This puts Europe and other countries in the world at risk of the emergence of other outbreaks ${ }^{13}$. Besides the provision of vaccinations, pharmacists help provide these vaccinations while informing the community on the dangers of the failure to carry out routine vaccination programs for children and other people with chronic illnesses.

When carrying out their businesses, pharmacists adhere to the protocols and guidelines given by the world international health bodies such as the World Health Organization and the Center for Disease Control. Pharmacists provide critical information to patients on where they can access vaccinations. Pharmacists play a vital role in the mobilization of members of the community during pandemics. They also help keep the community members with information on the need for other eradicated diseases. As mentioned earlier, during pandemics, access to routine vaccinations is disrupted by the pandemic ${ }^{14}$. According to the American Academy of Pediatrics, there is a need to maintain vaccination schedules for eradicated communicable diseases to prevent their resurgence.

Pharmacists in Europe have a long history of patient vaccinations and immunization. However, there exist limits on the age groups and types of vaccines that these groups can receive. There is a high level of uncertainty during pandemics, especially regarding communication, the flow of information, and vaccinations ${ }^{15}$. The pharmacists are allowed to carry out immunization programs; members of the community can access the vaccinations at a cheap cost and more convenience. Pharmacists can also provide information on outbreak resurgence to public health government authorities for actions. The role of pharmacists in vaccination programs can be direct through administering the vaccination themselves or indirectly through provision of vaccination information to the public members and giving vaccination information feedback to the government health care agencies ${ }^{16}$. World health bodies such as the world health organizations and the Center for Disease Control recognize pharmacists as essential members of the healthcare system in Europe and the world, especially during pandemics.

Pharmacists need to exhibit high levels of diligence and accountability when involved in vaccination programs during pandemics ${ }^{17}$. They should also ensure that they act in a proactive approach to curb future outbreaks. Pharmacists comprise valuable members of the healthcare sector prior to, during, and after the pandemic outbreak; they comprise the easily accessible healthcare provider directly or indirectly. The pharmacists can play an active role during pandemics by alleviating public concerns and worries by engaging the communities on prevention of transmission techniques where to seek vaccinations and other critical vaccination information.

\section{References}

1. BURSON R C, BUTTENHEIM A M, ARMSTRONG A, FEEMSTER K A (2016) Community pharmacies as sites of adult vaccination: A systematic review. Human vaccines \& immunotherapeutics, 12(12), 3146-3159.

\footnotetext{
${ }^{13}$ Japsen, B. (2020). Rite Aid: Pharmacist Role Will Expand Amid Coronavirus Outbreak.

${ }^{14}$ Mcelaney, P., Iyanaga, M., Monks, S., \& Michelson, E. (2019). The quick and dirty: a tetanus case report. Clinical practice and cases in emergency medicine, 3(1), 55.

${ }^{15}$ Meyers, R., Weiland, J., Holmes, A., Girotto, JE, \& Advocacy Committee on behalf of the Pediatric Pharmacy Advocacy Group. (2018). Position paper: pharmacists and childhood vaccines. The Journal of Pediatric Pharmacology and Therapeutics, 23(4), 343-346.

${ }^{16} \mathrm{Ou}, \mathrm{HT}$, \& Yang, YH K. (2020). Community pharmacists in Taiwan at the frontline against the novel coronavirus pandemic: gatekeepers for the rationing of personal protective equipment.

${ }^{17}$ Papastergiou, J., Folkins, C., Li, W., \& Zervas, J. (2014). Community pharmacist-administered influenza immunization improves patient access to vaccination. Canadian Pharmacists Journal/Revue des pharmaciens du Canada, 147(6), 359-365.
} 
2. CENTERS FOR DISEASE CONTROL AND PREVENTION (2007) Key facts about seasonal influenza (flu). The Influenza. http://www.CDC.gov/flu/key facts.htm (accessed November 28, 2008).

3. DROZD EM, MILLER L, JOHNSRUD M (2017) Impact of pharmacist immunization authority on seasonal influenza immunization rates across states. Clinical therapeutics, 39(8), 1563-1580.

4. EGGERTSON L (2010) Lancet retracts 12year-old article linking autism to MMR vaccines. Canadian Medical Association. Journal, 182(4), E199.

5. GUERCI J, CAMPBELL CT, CURTIS SD (2020) The Pharmacists' Role in Disease Outbreaks: Navigating the Dynamics of Uncertainty Before, During, and After Disease Outbreaks.

6. GUERCI J, CAMPBELL CT, CURTIS SD (2019) The Pharmacists' Role in Disease Outbreaks: Navigating the Dynamics of Uncertainty Before, During, and After Disease Outbreaks.

7. HOFFMAN J (2020) Vaccine rates drop dangerously as parents avoid doctor's visits.

8. HUSSAIN A, ALI S, AHMED M, HUSSAIN S (2018) The anti-vaccination movement: a regression in modern medicine. Cureus, 10(7).

9. ISENOR JE, EDWARDS NE (2019) The quick and dirty: a tetanus case report. Clinical practice and cases in emergency medicine, 3(1), 55.

10. MEYERS R, WEILAND J, HOLMES A, GIROTTO JE, ADVOCACY COMMITTEE ON BEHALF OF THE PEDIATRIC PHARMACY ADVOCACY GROUP (2018) Position paper: pharmacists and childhood vaccines. The Journal of Pediatric Pharmacology and Therapeutics, 23(4), 343-346.

11. OMECENE NE, PATTERSON JA, BUCHEIT JD, ANDERSOT AN, ROGERS D, GOODE JV, CALDAS LM (2019). Implementation of pharmacist-administered pediatric vaccines in the United States: major barriers and potential solutions for the outpatient setting. Pharmacy Practice (Granada), $17(2)$.

12. OU HT, YANG YHK (2020) Community pharmacists in Taiwan at the frontline against the novel coronavirus pandemic: gatekeepers for the rationing of personal protective equipment.

13. PAPASTERGIOU J, FOLKINS C, LI W, ZERVAS J (2014) Community pharmacistadministered influenza immunization improves patient access to vaccination. Canadian Pharmacists Journal/Revue des pharmaciens du Canada, 147(6), 359-365.

14. PATEL M (2019) Increase in measles cases - United States, January 1-April 26, 2019. MMWR. Morbidity and mortality weekly report, 68 .

15. RUBIN LG, LEVIN MJ, LJUNGMAN P, DAVIES EG, AVERY R, TOMBLYN M, KANG I (2014) 2013 IDSA clinical practice guideline for vaccination of the immunocompromised host. Clinical infectious diseases, 58(3), e44-e100.

16. SANTOLI JM (2020) Effects of the COVID19 pandemic on routine pediatric vaccine ordering and administration - United States, 2020. MMWR. Morbidity and mortality weekly report, 69.

17. TSUYUKI RT, BEAHM NP, OKADA H, AL HAMARNEH YN (2018) Pharmacists as accessible primary health care providers: review of the evidence. Canadian Pharmacists Journal/Revue des pharmaciens du Canada, 151(1), 4-5. 